\title{
The strip detector of the PANDA MVD
}

\author{
Tommaso Quagli*ł, Kai-Thomas Brinkmann, Robert Schnell and Hans-Georg \\ Zaunick \\ II. Physikalisches Institut, JLU Gießen \\ E-mail: tommaso.quagli@exp2.physik.uni-giessen.de
}

\begin{abstract}
PANDA is a key experiment of the future FAIR facility, under construction in Darmstadt, Germany. It will study the collisions between an antiproton beam and a fixed proton or nuclear target. The Micro Vertex Detector (MVD) is the innermost detector of the apparatus and is composed of four concentric barrels and six forward disks, instrumented with silicon hybrid pixel and doublesided silicon microstrip detectors; its main task is the identification of primary and secondary vertices. The main requirements include high spatial and time resolution, trigger-less readout with high rate capability, good radiation tolerance and low material budget.

The design of the strip barrel detector and the related recent developments are summarized in this document. Prototypes of double-sided strip sensors were characterized with a probe station and with proton and neutron irradiations. The sensors will be read out with a self-triggering ASIC, employing the Time-over-Threshold technique for energy loss measurement. The sensors and readout chips will be supported by 46 carbon fiber staves, embedding an active cooling system; a flexible multilayer bus will be used to route the signals on the stave towards the DAQ system. $\ddagger$
\end{abstract}

11th International Conference on Large Scale Applications and Radiation Hardness of Semiconductor Detectors

3-5 July 2013

Florence, Italy

\footnotetext{
* Speaker.

${ }^{\dagger}$ Supported by HIC for FAIR through HGS-HIRe.

${ }^{\ddagger}$ This work was partially supported by BMBF (grant no. 06 BN 9005 U and no. 06 GI 7007 I) and Helmholtz Graduate School for Hadron and Ion Research.
} 


\section{Introduction}

The PANDA experiment [1] is one of the key projects at the future FAIR facility [2]. The experiment will make use of antiproton beams colliding with a hydrogen or gaseous nuclear (e.g. $\mathrm{He}, \mathrm{Xe}$ ) fixed target to study the strong interaction at intermediate energies. The main topics of the $\bar{P}$ ANDA physics program are the spectroscopy of charmonium and open charm states and the search for exotic states [3].

The PANDA detector is composed of two parts: a target spectrometer around the interaction region and a forward spectrometer. The detector is designed to achieve an almost complete coverage of the solid angle and consists of several detectors for tracking, calorimetry and particle identification.

\section{MVD Design}

The Micro-Vertex-Detector (MVD) is the innermost component of the tracker system of the experiment [4]. Its main tasks are the reconstruction of the primary interaction vertex and of the secondary vertices from the decays of short-lived particles.

The layout of the MVD is conceived to provide a minimum of four points per track in the polar angle range between $9^{\circ}$ and $145^{\circ}$. The main detector requirements include high spatial $(<100 \mu \mathrm{m})$ and time ( $\sim 1.8 \mathrm{~ns} \mathrm{rms}$ ) resolution, high rate capability (up to $2 \cdot 10^{7} \overline{\mathrm{p}}$ annihilations per second), radiation tolerance (up to $10^{14} \mathrm{n}_{1} \mathrm{MeV}_{\text {eq }} / \mathrm{cm}^{2}$ ) and low material budget (below $10 \% \mathrm{X}_{0}$ ).

The MVD is divided into a central part with four concentric barrels and a forward part with six disks. A schematic view of the detector is shown in figure 1. The interface between the disk and the barrel part is located at a polar angle of approximately $40^{\circ}$.
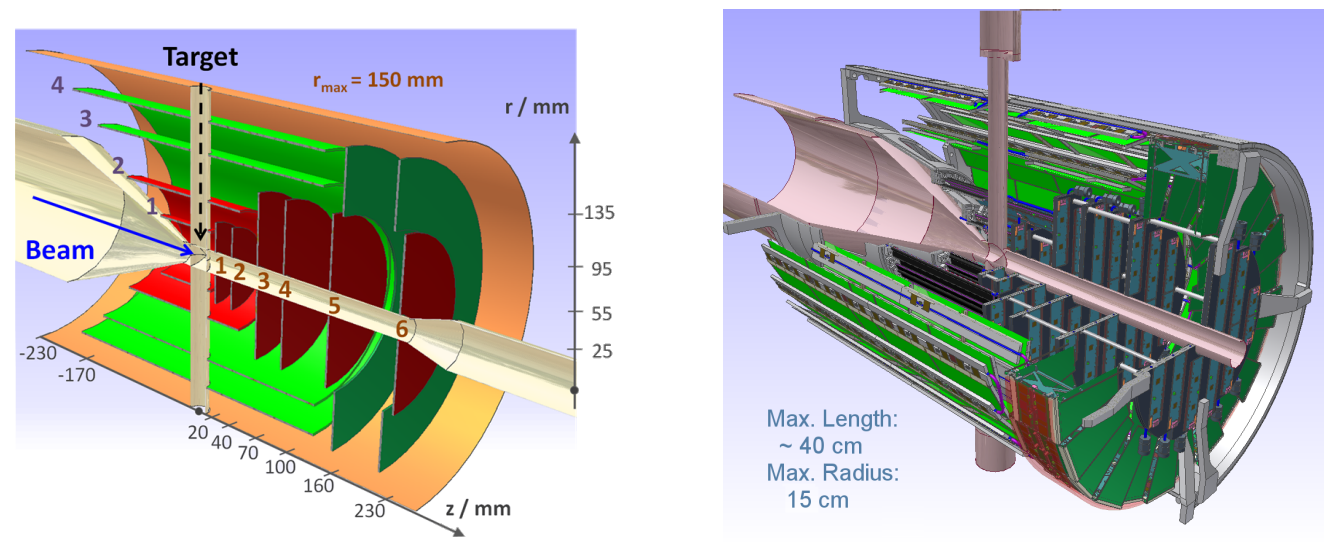

Figure 1: The $\bar{P} A N D A$ Micro-Vertex-Detector [4]. Left: Schematic layout; the red elements represent the pixel part of the detector, while the green components represent the strip part. Right: CAD drawing of the detector.

In order to cope with the high particle rate near the interaction region, the two innermost barrels and most of the disks are covered with hybrid pixel detectors, composed of epitaxial silicon sensors featuring $100 \times 100 \mathrm{\mu m}^{2}$ pixels and a trigger-less readout ASIC called ToPix [5]

The two outer barrels and part of the last two disks, which have less stringent rate requirements, use double-sided silicon microstrip detectors. 


\section{Strip detector design}

\subsection{Strip Barrels}

In the barrel part of the strip detectors the basic building block is a linear stave, featuring 4 to 6 sensors and the front-end electronics necessary to read out them. The barrels are formed by a paddle-wheel arrangement of the staves. 20 staves form the inner barrel, placed at a radius of $92 \mathrm{~mm}$, while the outer barrel, with a radius of $125 \mathrm{~mm}$, is composed of 26 staves (figure 2 right).

The sensors for the barrel part are double-sided silicon microstrips and come in two different shapes: rectangular $\left(60 \times 35 \mathrm{~mm}^{2}\right.$ for $896 \times 512$ strips $)$ and squared $\left(35 \times 35 \mathrm{~mm}^{2}\right.$ for $512 \times 512$ strips). The stereo angle is in both cases $90^{\circ}$ and the strip pitch is $65 \mu \mathrm{m}$. It is foreseen to read out every second strip of the sensors leaving a floating intermediate strip, thus obtaining a readout pitch of $130 \mu \mathrm{m}$; this has a tolerable impact on the spatial resolution, reducing at the same time by a factor of two the number of required front-end chips and electrical power [6].

The core of each stave is a composite support structure which embeds the cooling system for the readout electronics. Two adjacent staves share the same cooling pipe, thus creating a loop that avoids to have pipe connectors in the downstream region. The design of two staves with the position of the components is shown in the left part of figure 2.
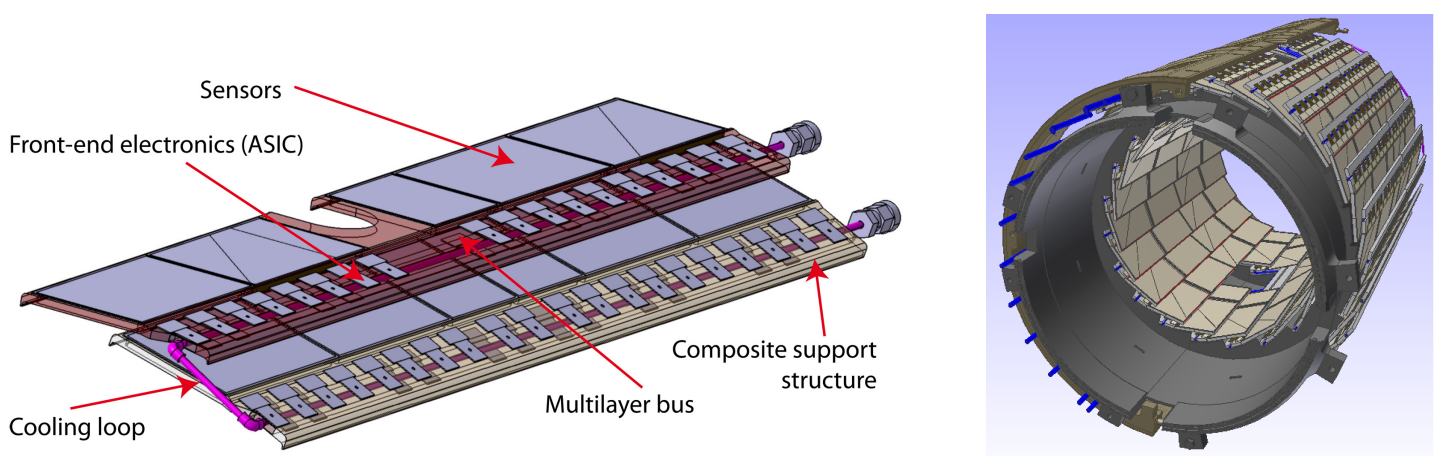

Figure 2: Left: Schematic design of two adjacent staves; the composite support structure with the embedded cooling system, the sensors, the readout electronics and the multilayer bus are visible. Right: design of the two strip barrels.

The sensors are placed on one side of the stave; the readout electronics is placed alongside of the sensor, on both sides of the stave in correspondence with the cooling pipe. A multilayer flexible bus is used to connect the sensor to the chips. The readout of the short strips, perpendicular to the stave, is performed directly at the side of the sensor, while the readout of the long strips, parallel to the stave, requires another bus to route the signals away from the sensor.

A detail of the cross section of one stave is shown in figure 3.

The stave is a sandwich structure of carbon fiber (type M55J, 200 um thick) and foam (Rohacell, $2 \mathrm{~mm}$ thick). The power dissipation (up to $18 \mathrm{~W}$ per stave) requires an active cooling system, which is implemented by means of an embedded nickel-cobalt alloy pipe. The pipe has a diameter of $2 \mathrm{~mm}$ and a wall thickness of $80 \mu \mathrm{m}$; the system uses water as cooling fluid at an input temperature of $18^{\circ} \mathrm{C}$. The chips are placed directly above the cooling pipe; in the region of the stave around 


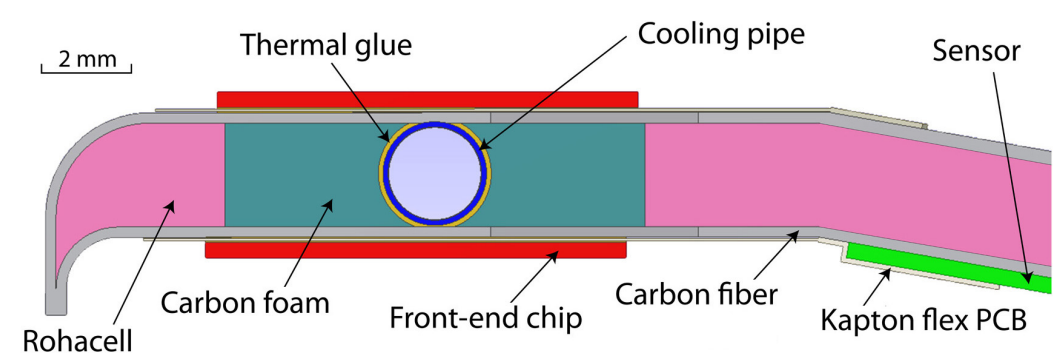

Figure 3: Detail of the cross section of a stave.

the pipe the Rohacell is replaced with a highly thermal conductive carbon foam (Poco HTC ${ }^{1}$ ).

The staves feature large cutouts in correspondence with the sensors, in order to reduce the material budget. A special design with a missing sensor is required for the top/bottom staves of both barrels around the target pipe. Six different stave layouts are needed for the whole strip detector. Figure 4 shows some preliminary prototypes of the staves.
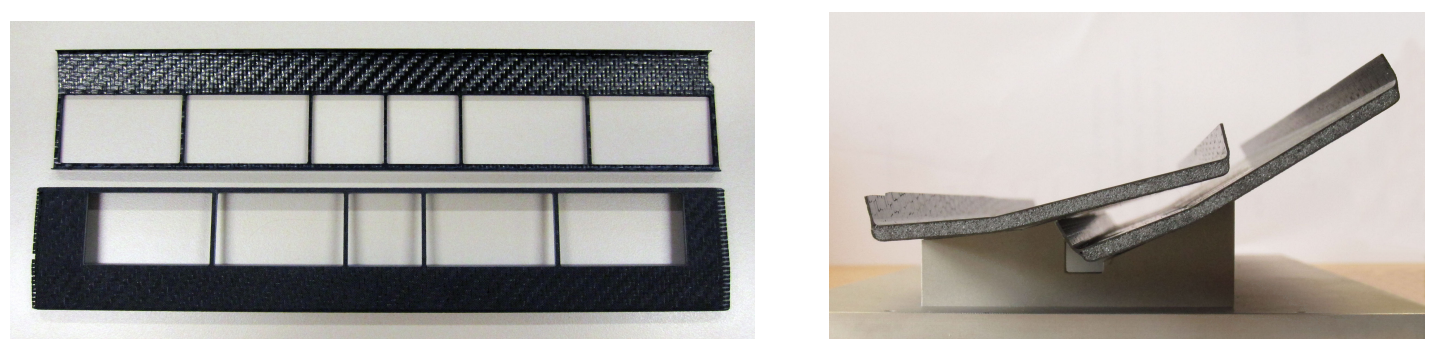

Figure 4: Left: prototypes of two composite staves for the outer and inner barrel (top and bottom, respectively). Right: cross section and relative position of two adjacent staves. The paddle-wheel arrangement allows for a small overlap of the sensors. The cooling system is not implemented in these preliminary prototypes.

\subsection{Strip Disks}

The disk part of the strip detector consists of two rings of trapezoidal double-sided microstrip sensors, placed around the last two pixel disks. Each sensor features 512 strips per side with a stereo angle of $15^{\circ}$ and a strip pitch of $67.5 \mu \mathrm{m}$. A drawing of a sensor is shown in figure 5 .

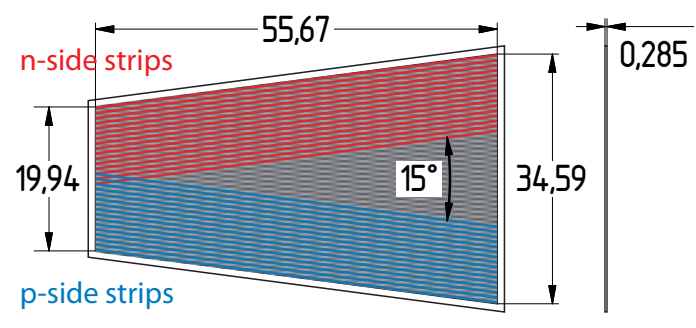

Figure 5: Geometry of a disk sensor; all figures are in $\mathrm{mm}$. The strip pitch is $67.5 \mu \mathrm{m}$; each side features 512 strips.

\footnotetext{
${ }^{1}$ Poco Graphite Inc. Poco htc. http://www.poco.com/
} 
The two rings are composed of 24 sensors each and are placed $155 \mathrm{~mm}$ and $215 \mathrm{~mm}$ downstream from the interaction point, respectively. Two corresponding sensors on the two disks compose a supermodule, as shown in figure 6.
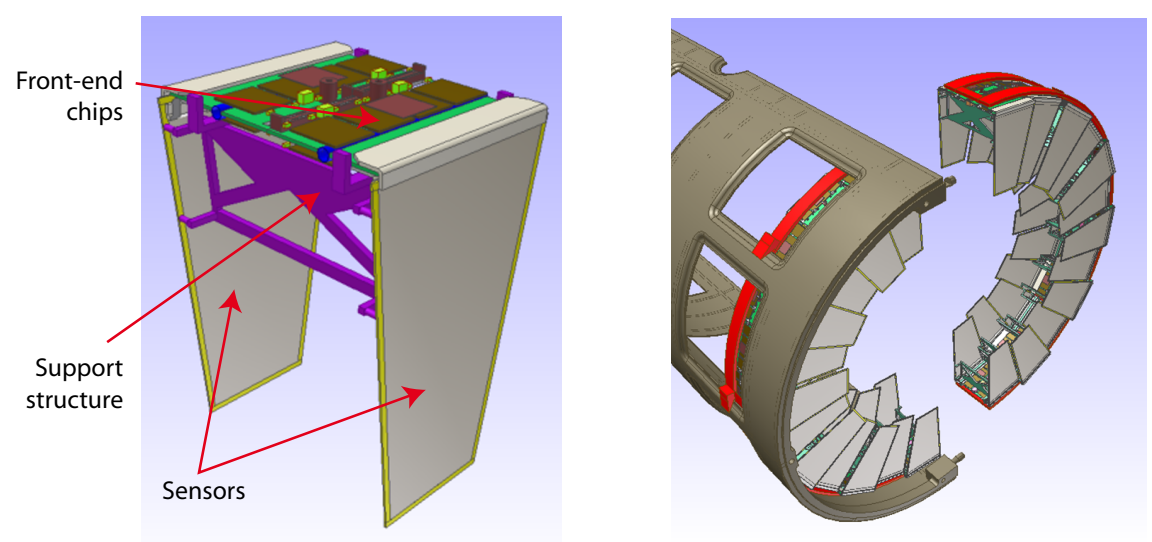

Figure 6: Left: sketch of a strip disk supermodule. Right: design of the strip disks of the MVD.

The two sensors are supported by a common carbon fiber structure; the front-end electronics is placed on a multilayer bus on the top of the supermodule, where the readout of the strips is performed.

\section{Double-sided silicon microstrip sensors}

The first batch of full-size prototype sensors for the barrel part of the MVD was produced in 2011 by CiS, Erfurt, Germany ${ }^{2}$. Each of the 4 " wafers contains two full-size prototypes (one square and one rectangular), another square sensor (S3) with an active area of $19 \times 19 \mathrm{~mm}^{2}$ compatible with the preexisting readout architecture [7] and five "Baby" sensors with a size of $8 \times 8 \mathrm{~mm}^{2}$. The material is an n-type Floating-Zone (FZ) silicon with resistivity between 2.3 and $5.0 \mathrm{k} \Omega \cdot \mathrm{cm}$; the wafers are $285 \mu \mathrm{m}$ thick. A photo of the wafer and details of the corners of a sensor are shown in figure 7 .
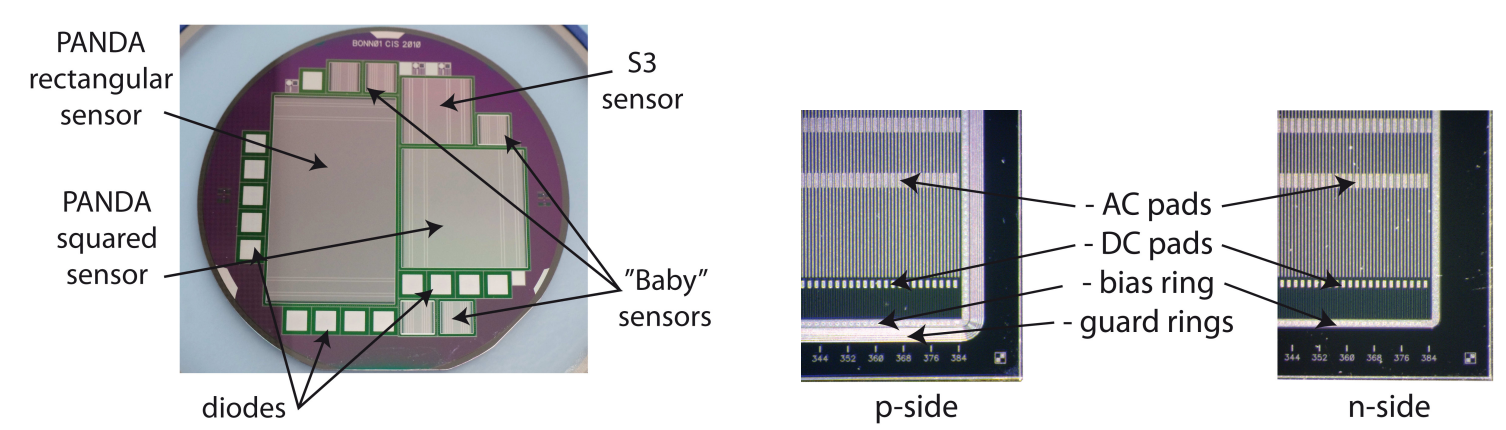

Figure 7: Left: photo of the 4" wafer with the full-size prototype sensors and the smaller structures. Right: corners of a prototype sensor [8].

${ }^{2}$ CiS Forschungsinstitut für Mikrosensorik und Photovoltaik GmbH. http://www.cismst.de 
The strip implants are $\mathrm{p}^{+}$-in-n on the front side and $\mathrm{n}^{+}$-in- $\mathrm{n}$ on the back side. The biasing of the strips is achieved with the punch-through technique; a p-spray implant is used to isolate the $\mathrm{n}$-side strips. Each strip features one DC pad and four AC pads to perform the readout, to allow for more freedom in the placement of the wire bondings in the prototyping phase.

The sensors were characterized using a probe station setup and a fixed-contact probe card on which a sensor was glued and connected by means of wire bonding. The measuring setup comprised an LCR-Meter Sourcetronic ST2819 and a high voltage power supply Iseg SHQ 124; the device under test was connected through a bias box which insulated the LCR-Meter inputs from the high voltage supply. The use of the probe card allows to connect all the strips on both the front and the back side of the sensor, offering the possibility to perform a thorough characterization of the device under test; on the other hand, it is a destructive measurement since the sensor cannot be reused afterwards. Two full-size rectangular sensors were characterized with the probe station and with the probe card, respectively [6, 8]. Additionally, a leakage current measurement was performed by the manufacturer on all delivered sensors; all the samples but two show a leakage current between 1 and $5 \mu \mathrm{A}$ for a wide range of reverse bias voltages, as shown in figure 8 .

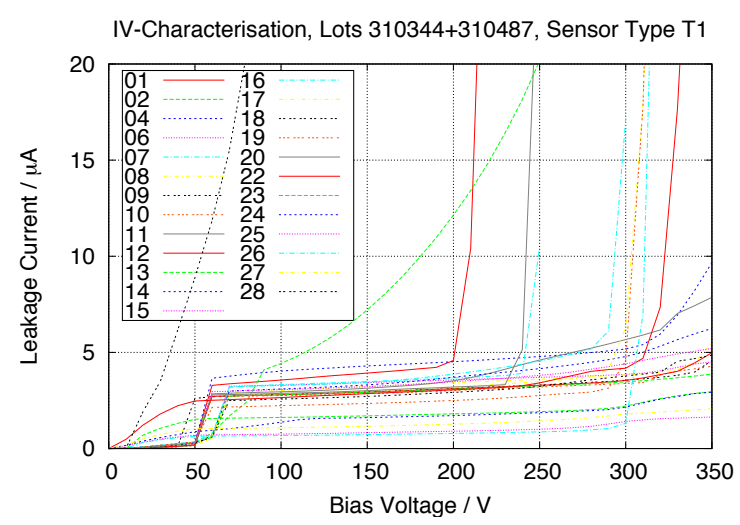

Figure 8: Leakage current behavior of $25 \overline{\mathrm{P}} \mathrm{ANDA}$ rectangular sensors [4].

Performed measurements include the leakage current, the full depletion voltage and the bulk, interstrip and coupling capacitances [6]. A selection of results of the characterization of a rectangular prototype sensor is shown in figure 9 and in table 1.
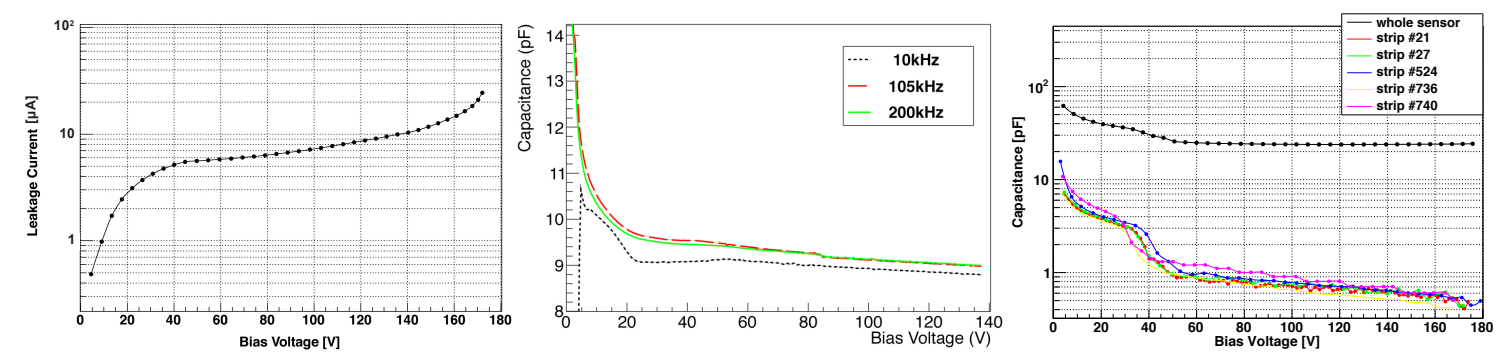

Figure 9: Characterization of a PANDA rectangular sensor. Left: Leakage current. Center: Interstrip capacitance. Right: Strip-to-bulk capacitance [8]. 
Table 1: Some parameters from the characterization of $\bar{P}$ ANDA sensors. The bulk capacitance requires the use of the probe card and was therefore measured only on one rectangular sensor; the coupling capacitance was measured on two rectangular and one "Baby" sensors; all other measurements were performed on two rectangular sensors.

\begin{tabular}{|c|c|}
\hline Full depletion voltage & $(50 \pm 5) \mathrm{V}$ \\
\hline Leakage current & $\sim 6 \mu \mathrm{A} @ 50 \mathrm{~V}$ \\
\hline Bulk capacitance & $(683.5 \pm 1.5) \mathrm{pF}$ \\
\hline Interstrip capacitance & $\sim 10 \mathrm{pF} \mathrm{@50} \mathrm{V}$ \\
\hline Coupling capacitance & $(54.0 \pm 1.5) \mathrm{pF} / \mathrm{cm}$ (p-side) \\
& $(46.3 \pm 0.9) \mathrm{pF} / \mathrm{cm}(\mathrm{n}$-side $)$ \\
\hline $\mathrm{C}_{\text {input }}$ & $(9.8 \pm 0.2) \mathrm{pF}(\mathrm{p}$-side $)$ \\
& $(17.1 \pm 0.4) \mathrm{pF}(\mathrm{n}$-side $)$ \\
\hline
\end{tabular}

The radiation hardness of the sensors was studied via irradiation of seven small "Baby" sensors with $14 \mathrm{MeV}$ protons at the Bonn Isochronous Cyclotron, applying fluences between $10^{13}$ $\mathrm{n}_{1 \mathrm{MeV} \text { eq }} / \mathrm{cm}^{2}$ and $10^{15} \mathrm{n}_{1 \mathrm{MeVeq}} / \mathrm{cm}^{2}$. Two additional neutron irradiations were performed with an AmBe neutron source (fluence $3 \cdot 10^{10} \mathrm{n}_{1 \mathrm{MeVeq}} / \mathrm{cm}^{2}$ ) and at the research reactor at the Delft University (fluence $1.6 \cdot 10^{16} \mathrm{n}_{1 \mathrm{MeVeq}} / \mathrm{cm}^{2}$ ). The irradiations were followed by an annealing phase of 80 minutes at $60^{\circ} \mathrm{C}$. The variation of the leakage current and of the full depletion voltage with the applied fluence are shown in figure 10 . The full depletion voltage remains below $100 \mathrm{~V}$ up to the fluence corresponding to the expected $\bar{P} A N D A$ lifetime $\left(10^{14} \mathrm{n}_{1 \mathrm{MeVeq}} / \mathrm{cm}^{2}\right)$.
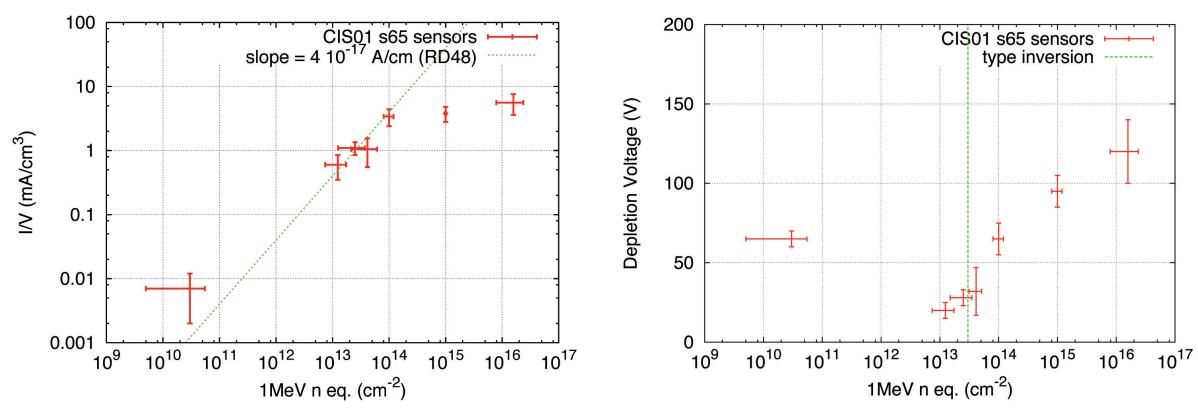

Figure 10: Leakage current and full depletion voltage of the irradiated sensors. The fluence corresponding to the type inversion, as evaluated by the RD48 collaboration in [9], is shown.

\section{Readout electronics}

The readout of the strip detector poses significant challenges. Since the experiment will not have a hardware trigger, the front-end must be able to run trigger-less and to sustain a rate up to $1 \mathrm{kHz}$ per channel. As derived from the characterization of the sensors, the chip must comply with an input capacitance in the order of $\sim 20 \mathrm{pF}$. The electronics must be radiation hard up to about $10 \mathrm{Mrad}$; additionally, in order to limit the material budget of the cooling system, the power consumption is limited to $\sim 1 \mathrm{~W} / \mathrm{cm}^{2}$. Finally, the ability to perform a charge measurement is a desirable feature. 
The lack of a front-end chip which met these requirements led to the choice of a custom development. The new ASIC is developed at INFN Torino in collaboration with JLU Gießen and FZ Jülich. The front-end is based on the TOFPET chip, an ASIC designed for the readout of Silicon Photomultipliers in a Time-of-Flight PET application [10]. The amplitude measurement is performed via the Time-over-Threshold (ToT) technique. The time digitization will be performed with TDCs with analog interpolators with a time binning of $50 \mathrm{ps}$, adapted from the TOFPET chip [10]. The submission of the first chip prototype is expected in 2014.

\subsection{Strip detector powering}

The powering system of the strip detector must provide low voltage power supply to the readout chips and to the Module Data Concentrators (MDC) and uses DC-DC converters. Since at least part of the DC-DC converters will operate inside the $2 \mathrm{~T}$ solenoidal magnetic field of the target spectrometer, the use of ferrite-based converters is excluded. The evaluation of converters based on air cores, developed at CERN for LHC upgrades, is ongoing.

The design foresees a total of 296 sensors, read out by $\sim 3300$ front-end chips. The smallest electrically independent module is composed of one sensor with the related readout chips and one MDC. Each sensor requires five power domains (four for the analog and digital supply of the p-side and n-side chips, respectively, plus one for the MDC), for a total of 1500 DC-DC converters.

The converters needed for the strip detector will be placed around the beam pipe upstream from the MVD and connected to the detector modules via aluminum cables, in order to reduce the material budget. The number of power domains per stave leads to a large number of cables that have to be brought inside the sensitive volume of the detector. Additionally, because of the asymmetry in the design of the MVD, the cables can be routed only in the backwards direction: the routing of services will be therefore a crucial issue in the detector design.

\section{Multilayer bus}

The current stave design foresees a multilayer flexible bus to connect the sensors to the chips. The material chosen for the bus is a copper-clad polyimide film manufactured by Thinflex ${ }^{3}$, which features two copper layers $12 \mu \mathrm{m}$ thick laminated on a $50 \mu \mathrm{m}$ kapton substrate. The copper layers are etched in the desired layout and covered with a protective varnish, for a total thickness of about $100 \mu \mathrm{m}$.

A first prototype of the bus, designed to read out one small strip sensor with one APV25 readout chip, was produced by the company Swiss $\mathrm{PCB}^{4}$ and is currently under test. The flex features an integrated fan-in structure to route the signals and to adapt the pitch from the $44 \mathrm{\mu m}$ of the staggered pads the front-end of the chip to the $150 \mu \mathrm{m}$ on the sensor. The sensor is a small S3 prototype, described in section 4, designed to be compatible with the preexisting readout architecture. Its pitch, although slightly larger than the one to be used in the final design, was chosen in order to read out the entire sensor with just one chip. The lines for the input/output and powering of the chip and for the biasing of the sensor are also integrated on the hybrid PCB. In figure 11 the bus prototype and its test assembly are shown.

\footnotetext{
${ }^{3}$ Thinflex Corporation. http://www.thinflex.com.tw

${ }^{4}$ GS Swiss PCB AG. http://www.swisspcb.ch
} 

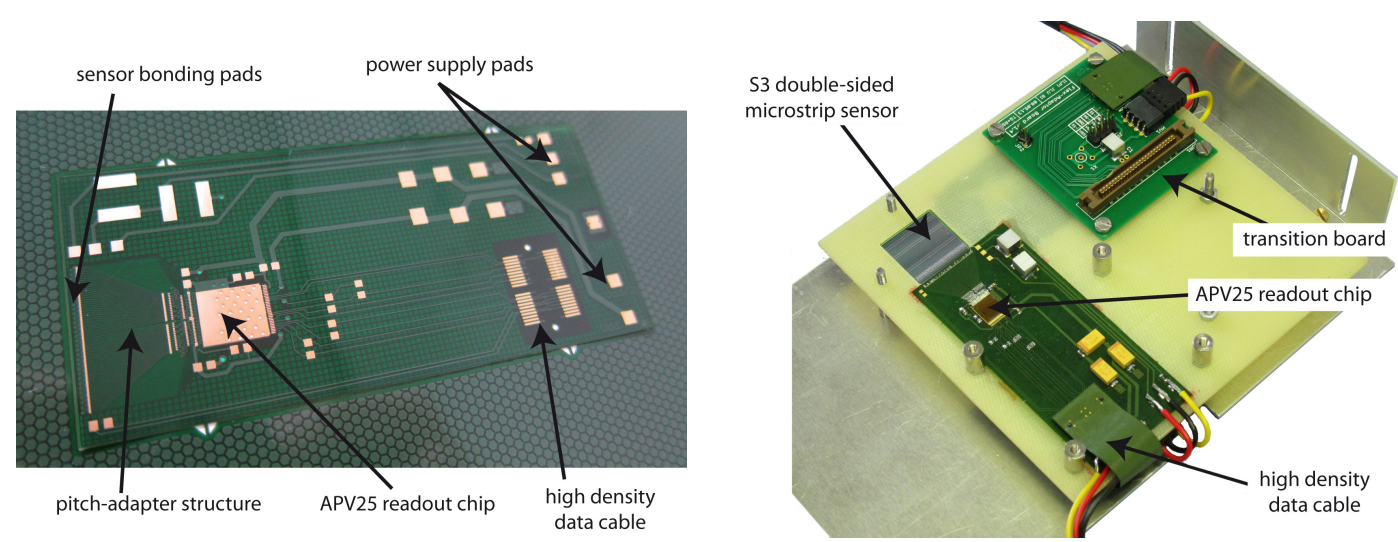

Figure 11: Left: prototype of the flexible multilayer bus. Right: test assembly. The readout of the doublesided sensor is performed with two identical busses, placed on both sides of a glassfiber plate. A transition board is used to provide the low voltage power supply and the sensor biasing and to convert the high-density data cable to a standard compatible with the preexisting readout architecture.

\section{References}

[1] $\bar{P} A N D A$ Collaboration, Letter of Intent for: PANDA: Strong Interaction Studies with Antiprotons, 2004.

[2] GSI Helmholtzzentrum für Schwerionenforschung. FAIR - An International Accelerator Facility for Beams of Ions and Antiprotons. Baseline technical report, 2006.

[3] $\bar{P} A N D A$ Collaboration, Physics Performance Report for PANDA: Strong Interaction Studies with Antiprotons, 2009, [arXiv:0903.3905].

[4] The PANDA Collaboration, Technical Design Report for the: PANDA Micro Vertex Detector, [arXiv:1207.6581v2], 2011.

[5] L. Zotti et al., Hybrid Pixels for the Micro-Vertex Detector of the PANDA Experiment, PoS (Bormio2012) 020

[6] H.-G. Zaunick, Developments toward a Silicon Strip Tracker for the PANDA Experiment, PhD Thesis, Universität Bonn, 2012

[7] R. Schnell et al., FPGA-based readout for double-sided silicon strip detectors, JINST 6 (2011) C01008.

[8] T. Quagli et al., Development of Silicon Strip Sensors and radiation hardness studies for the PANDA MVD, IEEE NSS CR 2012, N14(219) 1365-1369

[9] ROSE Collaboration (RD48), Third RD48 Status Report, Dec. 1999. Available online at http://rd48.web.cern.ch/RD48/

[10] M.D. Rolo et al., TOFPET ASIC for PET applications, JINST 8 (2013) C02050 